\title{
Aprendizaje infantil y ethos lúdico
}

\author{
Desirée López de Maturana L. \\ Vicepresidenta Ejecutiva de la Junta Nacional de Jardines Infantiles, Santiago, Chile. \\ Email: desireelopezdem@yahoo.es
}

\begin{abstract}
Resumen: ${ }^{1}$ Observar la construcción y la reorganización permanente de los juegos creados e imaginados por los niños, es una experiencia interesante que provoca profundas reflexiones respecto de nuestra propia práctica docente, particularmente, en la educación inicial. La inmensidad del espacio y el tiempo que conforman el contexto de juego, los usos, las ensoñaciones, las ideas y el esfuerzo que cada niño pone en este fluir de ideas, le otorgan sentidos profundos a estas experiencias personales y un gran valor intersubjetivo cuyo arraigo colectivo es un acto existencial con el cual los niños se comprometen. En un intento por caracterizar o describir de forma genuina estas experiencias, surge la denominación de "ethos lúdico”, cuyo valor conceptual abre ventanas para observar más de cerca los elementos o dimensiones axiológicas de convivencialidad, y de construcción cultural con que los niños acceden y aportan a la generación de su propio conocimiento. Entre estos aspectos que lo definen y relevantes a la hora de pensar en cómo hacer para favorecer sus aprendizajes en las escuelas, podemos mencionar que los espacios y tiempos lúdicos son indeterminados y complejos; es decir, sus acciones son determinadas por reglas constitutivas emergentes y dinámicas que obedecen a una secuencia accional, por lo que tienen un sentido y un significado contextual.
\end{abstract}

Palabras clave: Ethos lúdico, aprendizaje situado, contextualismo, autoorganización

\section{Childhood learning and playful ethos}

\begin{abstract}
Watching the construction and permanent reorganization of games created and imagined by children, is an interesting experience that generates profound thoughts about our own teaching practice and particularly on the early stages of formal education. The vastness of space and time that shape the game context, the different uses, dreams, ideas and effort that each child places in this flow of ideas, gives deeper meaning to these personal experiences and great intersubjective value, whose collective rooting is an existential act with which children commit. In an attempt to characterize in the most genuine way these experiences, arises the name "playful ethos", whose linguistic connotation opens windows to look more closely at the elements or axiological dimensions of conviviality and cultural construction with which children access and contribute to the generation of their own knowledge. Among the aspects that define this concept, and relevant at the moment of thinking in how to favor their learning processes at school, we can mention that playful time and space are undetermined and complex; this is, their actions are determined by constitutive and dynamic emerging rules that obey to an actional sequence, and thus have a contextual sense and meaning.
\end{abstract} organization

Key words: Playful ethos, situated learning, contextualism, self- 


\section{Educação Infantil e ethos lúdico}

Resumo: Observar a construção e reorganização permanente das brincadeiras criadas e imaginadas pelas crianças é uma experiência interessante que provoca profundas reflexões sobre nossas próprias práticas de ensino, especialmente na educação infantil. A vastidão do espaço e do tempo que fazem o contexto do jogo, usos, sonhos, idéias e esforço que cada criança coloca neste fluxo de idéias, dão significados mais profundos a essas experiências pessoais e de grande valor intersubjetivo cujas raízes coletivas representam um ato existencial com que as crianças se comprometem. Na tentativa de caracterizar ou descrever genuinamente essas experiências, surge o termo "ethos lúdico”, cujo valor conceitual abre janelas para olhar mais de perto os elementos ou dimensões axiológicas de convívio e de construção cultural com que as crianças acedem e contribuem para a geração do seu próprio conhecimento. Entre os aspectos que o definem e são relevantes na hora de pensar em como continuar a sua aprendizagem nas escolas, podemos mencionar que os espaços e momentos de lazer são indeterminados e complexos; ou seja, suas ações são determinadas por regras constitutivas emergentes e dinâmicas que obedecem a uma seqüência acional, razão pela qual eles têm um sentido e um significado contextual.

Palavras-chave: Ethos lúdico - aprendizagem situada - contextualismo auto-organização.

$* * *$

Bateson (1998) en su estudio antropológico realizado específicamente con la cultura iatmul en Bali, incorporó la noción de "ethos", como un instrumento conceptual útil para comprender con mayor agudeza la cultura. Lo define como la expresión de un sistema culturalmente estandarizado de organización de los instintos y emociones de los individuos, que se sustenta en el análisis y la evidencia de ciertas recurrencias. Si bien la utilidad de este concepto para la investigación de Bateson no garantiza que también la tenga para otros estudios, coincidentemente, me surgió de manera natural, esta denominación mientras realizaba los registros de campo. Esto me permitió equilibrar la tendencia a interpretar sólo desde mi perspectiva experiencial y epistémica adulta, el comportamiento infantil.

Los niños (entiéndase en lo sucesivo, también niñas) nos muestran permanentemente, a través de sus juegos, diversas realidades que crean y que las viven intensamente. Lo interesante es que estos juegos surgen en una dimensión paradigmática y epistemológica propia de los niños, muchas veces invisibles a los ojos de los adultos, tal vez porque cancela la determinabilidad absoluta que lo hace pensable y comprensible; esta realidad sigue un orden estético que radica en el movimiento, en la figura, en lo bello, en el ritmo y en la armonía.

Maturana (2003) señala que, los niños de todo el mundo viven, a través de sus juegos rituales, que usan las mismas configuraciones de movimientos, que en el origen de la humanidad fueron el fundamento operacional en el desarrollo de la conciencia de sí, de la conciencia social, y de la conciencia de mundo, es decir que los juegos infantiles no son meras acti- 
vidades exentas de sentido, impuestas y exógenas al sujeto que juega, sino por el contrario, son acciones o funciones con significado para el jugador y que se traducen como factores de la vida cultural y, tal como dice Huizinga (1972), para profundizar en el concepto de juego debemos buscar justo allí, donde la biología y la psicología terminan su tarea.

En uno de los registros realizados en mi trabajo de campo, puse como ayuda memoria "zapping” para recordar la multirealidad o mundos paralelos que observaba mientras los niños jugaban; cambiaban los personajes, los roles, los escenarios de manera imperceptible para mí, en un fluir maravilloso. Con el tiempo, revisando bibliografía sobre visiones cuánticas respecto de los procesos sociales, fui incorporando otros elementos al análisis. Entendí que esta multirealidad no era posible abordarla a partir de fenómenos aislados sino a partir de interrelaciones, porque el comportamiento de una realidad está determinada por las conexiones que tiene con el conjunto y, puesto que es imposible saber con precisión cuáles son estas conexiones, tenía que modificar la visión clásica y parcial de causa y efecto por un concepto más amplio, más holístico. Para los sistemas cuánticos la no separabilidad implica que cualquier cambio en una de sus partes influenciará directamente sobre las demás ya que toda la realidad forma un todo indivisible (Sagan, 1983)

En este nuevo marco referencial se fue configurando la significación del ethos lúdico, cuyo marco epistemológico que en él se devela, nos interpela como educadores a erradicar los presupuestos con los que tradicionalmente intentamos y creemos comprender la lógica infantil, para dar un salto desde el determinismo al holismo, de la certeza a la incertidumbre, de lo absoluto a lo relativo, de lo separado a lo unido y de lo único a lo múltiple.

Osho hace alusión a las parábolas que sostienen que el hombre vivió una vez en el paraíso y luego, por alguna razón, fue expulsado de él, para referirse a que todo hombre, nace en el paraíso y después lo pierde. Pierde la inocencia, el asombro, la experiencia de la verdad y “de la hermosa danza de la vida en todas las cosas"; luego vive permanentemente buscando el paraíso que no está en otra parte, que en la propia infancia. Esta búsqueda se vuelve cada vez más infructífera, puesto que buscamos queriendo encontrar respuestas conceptuales de lo que no es conceptual, entonces vamos construyendo una verdad fragmentada, mal hecha a pedazos, pero posible de definir. Así nos vamos acomodando dentro de una coraza de saberes adultos que nos alejan cada vez más de la conciencia incondicionada de los niños.

En el siguiente registro, se describe una situación ocurrida en un jardín infantil, al término de la jornada diaria, cuando los niños se preparaban para regresar a sus casas. A este momento por lo general, no se le asigna valor educativo, porque no hay una planificación que oriente algún trabajo específico, ni se ha prescrito una "intención educativa”: 
La educadora dice: ¡bien hemos terminado!, pueden sacarse sus delantales para ir a casa.

Los niños se sacan sus cotonas, las niñas su delantales, (todos realizan solos esta actividad) dirigiéndose a la salita donde guardan sus pertenencias

José se devuelve al área de lectura y regresa intentando meterse unos cuentos en el bolsillo de su cotona desabotonada, donde no caben. Los deja sobre una mesa y se saca la cotona cuidando que no se los tomen. Se acerca Cristina e intenta tomar los cuentos, pero José reacciona rápidamente y le dice: "Cristi, anda a buscar más cuadernos (cuentos) para que esto sea una librería. Cristina no lo hace, pero él sigue con su idea y pone los cuatro cuentos sobre una mesa, ordenados en dos filas de dos [rápidamente el contexto comienza a cambiar].

Llega Pablo, que ha estado observando a la distancia y le dice: ¡hola! ¿tiene un libro donde salga una osa y un oso?, José - quien ahora está sentado en una silla- sin pararse contesta a la vez que muestra uno de los cuentos al "cliente", ¿éste señor? -iya bueno me lo voy a llevar!- responde Pablo hojeando el libro a la vez que agrega, ¿cuál más tiene?

Llega Catalina, observa la situación por un tiempo muy breve, se sienta en otra silla y con una voz un tanto quejumbrosa dice: "necesito un libro para mi hijo".

Llega Cristina, toma un cuento y Pablo con gesto de reprobación le dice: ¡no! Que esto es una librería.

Ignacio también se acerca y pregunta ¿puedo jugar o tengo que traer más libros de allá? [Indicando la repisa del área de lectura]. Los niños lo aceptan así, sin nuevos libros

Llega el papá de José a buscarlo. Rápidamente Catalina asume el rol de José y comienza a vender libros a cuatro pesos. Ella queda sola en la mesa, porque los demás se arreglan para la salida. Durante 5 minutos, Catalina ordena uno sobre otro los cuentos y los mira con dedicación y sigue sola pero siempre en su rol de vendedora de libros. Luego se acerca Ignacio y le dice: señora ¿me puede arrendar esto? indicando uno de los libros y haciendo un gesto de sacar dinero de su bolsillo...

Toda definición de juego dramático dirá, de una u otra forma, que durante estas creaciones de realidad, los niños enriquecen y aumentan su propia capacidad argumentativa, solucionan problemas, ensayan y fortalecen de manera individual y colectiva los distintos tipos de expresión oral, gestual, corporal, manifestando su propio bagaje experiencial, emocional y 
su conciencia de sí. En este registro encontramos que las propuestas de cada niño, son admitida y se contextualizan rápidamente, volviéndose un juego dramático por excelencia

También podemos observar, claramente, que cada propuesta o aporte emerge contextualmente sin necesidad de contar con un guión previo, porque quienes se van sumando incorporan su propuesta, su función, sus desplazamientos, su gestualidad, sus emociones y sus parlamentos, sin ningún tipo de instrucción, ni regla previa que demande, aparentemente, algún requisito para ello. Lo evidente es que todo aporte individual deviene en colectivo y fluye convirtiéndose en una experiencia autoorganizada, que en su relación dialéctica encuentra su dinamismo y su vigencia. Este espacio de vida paralelo, impenetrable para quienes no saben reconocer las claves esenciales de su naturaleza o de su estructura, es lo que he denominado "ethos lúdico”. Intentar definir o hacer una caracterización de este ethos, es una gran responsabilidad, por la tendencia a establecer un "deber ser”, que no haga justicia a la riqueza y la profundidad de los acontecimientos que ocurren en su centro.

\section{Sobre el “ethos lúdico” podemos decir que:}

- Es una verdadera morada donde la realidad cambia de manera insospechada; los niños viven y transitan por experiencias que van transformándose en historias que vitalizan ese nuevo mundo y develan elementos o dimensiones axiológicas de convivencialidad, y de construcción cultural. Cada registro se vuelve una narrativa elaborada colectivamente que nos muestra parte de ese mundo paralelo donde ellos como moradores, se relacionan y dan sentido, pero al que no podemos acceder si no compartimos este ethos que lo define.

Este conocimiento intersubjetivo que construye la experiencia lúdica es lo que lo configura como espacio habitable. Se percibe como un acto existencial con el cual los niños se comprometen, desarrollan su capacidad creativa para comunicar, representar y expresar la realidad a partir de la elaboración original que hacen desde sus sentimientos, ideas, experiencias y sensibilidad, recreando la realidad, con sentido estético, donde la relación entre las formalidades sociales, ya internalizadas y los nuevos contextos, van constituyendo una situación de aprendizaje tan completa, que les permite satisfacer necesidades personales, sociales, lúdicas y cognitivas, en un devenir incierto.

- Es un espacio sistémico, donde las acciones son convencionales, determinadas por reglas constitutivas emergentes: no están establecidas previamente, sino que ocurren a través de un proceso de auto-organización. En este proceso auto-organizativo, los niños asimilan el ambiente externo en el que se encuentran, desarrollan una idea original, que se va reinterpretando de acuerdo a nuevas exigencias que se satisfacen por la resignificación permanente de los elementos contextuales, logrando la adaptación. Así, el conocimiento se organiza en función de las exigencias pro- 
pias de los niños, y no por exigencias de orden externo, de allí que la posibilidad de introducir en el contexto de la educación formal, la sola reflexión respecto de este tema, aporta considerablemente al cambio y a la renovación de dichas estructuras, proporcionando una visión futura de diálogo entre los niveles de formalidad e informalidad, de institucionalidad y cotidianeidad, para comprender que las "instrucciones" externas no necesariamente estarán resonando significativamente en los procesos cognitivos internos de los niños.

Desde una óptica Vigostskiana, podemos decir que en estos espacios los niños son maestros en lo que teóricamente se ha denominado aprendizaje situado, toda vez que se entiende que en una actividad situada los conocimientos y el entorno están en íntima relación, por lo que no existe problema de descontextualización de los conocimientos, sino más bien aumenta la transferencia del saber al contexto, a la vez que este vuelve a poner nuevos desafíos aumentando el conocimiento, la solución de problemas y por supuesto, el aprendizaje.

- En su interior las reglas son propuestas por cualquier miembro del grupo. Como también, por lo general, los roles son autodesignados. Como ya se ha señalado, los niños van construyendo la experiencia a partir de una "idea" inicial, que surge de su relación circunstancial con el entorno. Se sumergen en esa realidad indeterminada, que carece de un guión pre-establecido, pero donde prevalecen las normas de cortesía y paradojalmente de orden. Los niños tienen un solo propósito común, jugar, entretenerse, incluso desplegar su esencia creativa para lo que no se requiere un diseño previo, porque la construcción colectiva es permanente y perfecta.

Si bien los niños, ponen al servicio de su juego creativo, todos los estímulos que perciben del entorno, están en una dimensión distinta, donde el orden lógico pasa a ser un orden histórico que les permite vivir al mismo tiempo el pasado y el futuro, transformando los elementos concretos de su entorno (objetos) en lo necesario para vivir genuinamente esa realidad imaginativa.

- Su configuración espacial y temporal, debe entenderse desde una noción de "decurso del tiempo", donde la irreversibilidad de los acontecimientos se comprende en el marco de las opciones, las probabilidades y las bifurcaciones que devienen en el fluir de los sucesos. (Spire, 2000), es decir, no corresponde a un tiempo lineal, cronológico, ni episódico; en este caso los niños se van sumando al juego con su aporte individual congruente con la realidad a la que ingresa, rápidamente entra también, en el fluir de acciones y acuerdos propios que van permitiendo la convivencia al interior de espacio de juego. Por esta misma razón, las reglas son dinámicas, tienen una secuencia accional, con sentido y significado contextual. Los niños son capaces de transitar por distintas dimensiones de espacio y tiempo, es decir por distintos “aquí” y distintos “ahora” que existen simultáneamente, todos igualmente reales desde su vivencia, por lo tanto las respuestas obedecen a la dimensión temporal y espacial que las contextualiza. 
Claramente, esto genera una brecha epistemológica entre adultos y niños, porque mientras los niños están absortos en una tarea, disfrutando en plenitud y prestando atención al proceso en sí mismo, los adultos estamos prestando atención a los resultados esperados empeñados en calzar en un estado de conciencia exógeno, de apariencia racional, "normal”. Es importante hacer explícita esta diferencia, para reencontrarse en ella y no generar una lucha inconveniente por subyugarse mutuamente.

- Su dinámica y su vigencia, necesita de las propuestas individuales que van rompiendo la monotonía, por ello ocurren permanentemente, y su aceptación radica en que el aporte de uno pasa a ser de todos. Las diversas situaciones que ese van sucediendo por estos aportes individuales, constituyen un todo sincrónico y holístico donde surgen elementos articuladores tan sutiles que son casi imperceptibles. Es más bien una construcción colectiva donde el aporte particular es lo que va conformando un todo dinámico, cuyo atractor pareciera ser, precisamente esa dialéctica permanente entre lo individual y lo colectivo. Efectivamente, desde una óptica externa, la secuencia del juego parece episódica, a veces desconectada, no obstante existe esta articulación, esta fuerza auto-organizativa subyacente que integra sinérgicamente los sucesos.

La teoría cuántica, nos entrega una nueva perspectiva para transformar nuestras nociones de relación, incorporando el concepto de ser, como un dualismo indeterminado de onda/partícula, junto con el concepto de movimiento que descansa en transiciones virtuales. Esta concepción por ende, comprende la realidad espacio temporal como aspectos múltiples de una totalidad mayor que a la vez las define y las significa cada vez. (Zohar, 1996)

Este indeterminismo es en sí, una instancia de aprendizaje y de socialización, desempeña un papel insustituible en los procesos educativos, por lo general "informales", que aceptan como factor esencial e imprescindible la complejidad y el caos. Lamentablemente, el juego utilizado en la escuela fluye reglamentado externamente y no a través del fluir de ideas imprevisibles y creativas de los niños. Lo dicho no trata de negar la importancia que tiene que los niños acepten normas externas, sino del hecho de privarlo del gozo de la búsqueda casual, ocasional y fortuita. La exploración, agudiza los sentidos, permite conocer y aprender activamente el entorno, y proporciona una visión futura de diálogo entre los distintos contextos educativos, cotidianos y formales. Estas realidades que emergen, nos llevan a explorar las conexiones metafóricas y las relaciones análogas que existen entre el pensamiento de los niños y el mundo, dado que, esto nos permite ver qué cosas aparentemente diferentes, son iguales

- Otra característica es que los juegos terminan cuando dejan de ser gozosos y entretenidos o por la intromisión de alguien ajeno.

Finalmente cabe hacer una síntesis para decir que los niños crean los juegos y a la vez estos se vuelven instancias de aprendizaje que ofrecen 
considerables posibilidades de funcionamiento que les van exigiendo poner en juego funciones mentales superiores como la abstracción, la representación, las anticipaciones, las combinaciones y las operatorias, para mantener su dinámica y su vigencia. Por cierto, los niños no temen a la incertidumbre, no necesitan de un plan pre- escrito, para desenvolverse con absoluta fluidez. Viven y construyen creativamente esta experiencia histórica por propia motivación. Es muy revelador ver como esta multiplicidad de vivencias sensoriales se ponen en contacto dentro del sistema del cerebro sobreponiendo varias y distintas nociones, en un indeterminismo que subyace a todo proceso de pensamiento, decisiones y creaciones.

La educación exige a los educadores(as) confiar en la autonomía de los niños, sin interferir, sólo estar atentos para cuando se arriesgan adentrándose en lo desconocido, en lo inexplorado. El temor es la causa de moldes y patrones rígidos de vida que imponemos a los niños, para dirigirlos por un camino determinado, hacia objetivos que paulatinamente van minando su originalidad y su condición de seres humanos en permanente autoconstrucción. Optar por enfoques humanistas coherentes y genuinos, es optar por una educación liberadora, que permite la plena realización y el desarrollo de su conciencia crítica, para suscitar su inconformidad con lo establecido y de “ser” ellos, en comunión con los otros y con el mundo.

Afortunadamente en los últimos años el tema de la niñez se ha puesto sobre el tapete en nuestro país, generándose recursos para apoyar y acompañar a los niños y las niñas y sus familias, a través de políticas públicas que se materializan en un sistema de protección social y de infancia. Todo ello es un gran salto desde el punto de vista social y económico, pero en absoluto ha resuelto de manera genuina la invisibilidad de los niños y las niñas reales; ciudadanos críticos, dinámicos, creativos y generadores de cultura, porque aún persevera la imagen del niño-adulto.

Poner atención al juego de los niños y descubrir su ethos, nos permite revalorizar la actividad lúdica como fuente de libertad y creatividad del ser humano, cuya connaturalidad le permite trascender como esencia y manifestarse adoptando diversas formas artísticas. Desde esta perspectiva naturalmente humana y creativa del juego, se rescata su valor epistemológico y la urgente consideración en la educación. Es imperativo reflexionar creativamente y descubrir nuevos modos de pensar aquello que ya se ha pensado, nos "empuja” a re-contextualizar constantemente nuestros modos cognitivos, a convertirnos en "brujos o brujas crea contextos”, para generar nuevos espacios de convivencia y de reflexión con los otros, como un acto de amor y creatividad, porque la transformación epistemológica dependerá del contexto desde el cual la reflexión emerge. (Bateson, 1982). 


\section{Nota}

${ }^{1}$ Este artículo se ha escrito a partir del trabajo realizado en el Proyecto FONDECYT 110577: "Asombros educativos infantiles y propensión a aprender". 


\section{Bibliografía}

Bateson, G. (1982), Naturaleza y Espíritu. Una unidad necesaria. Amorrortu, Buenos Aires.

Ídem (1998), Pasos hacia una ecología de la mente. Editorial. Lohlé-Lumen. Buenos Aires.

Capra, F (2003), Conexiones Ocultas. Barcelona: Anagrama.

Calvo, C. (2008), Del mapa escolar al territorio educativo. Disoñando la escuela desde la educación. Nueva Mirada Ediciones, $3^{\text {a }}$ edición. La Serena.

Dadamia, O. (2001), Educación y creatividad. Encuentro en el nuevo milenio. Editorial Magisterio. Buenos Aires.

García-Huidobro,V. (1996), Manual de Pedagogía teatral. Santiago de Chile: Editorial Los Andes.

Huizinga, J. (1972), Homo ludens. Alianza/Emecé. Madrid.

Marina, J.A. (2005), Aprender a vivir. Ariel S.A. Barcelona.

Maturana, H. (1990), Biología de la Cognición y Epistemología. Ed. Universidad de la Frontera. Temuco.

Ídem (2003), Amor y juego. Fundamentos olvidados de lo humano. Desde el patriarcado a la democracia. Ed. Comunicaciones Noreste Ltda. Santiago de Chile.

Ídem (2007), Transformación de la convivencia. Ed. Dolmen. Santiago de Chile.

Sagan, C. (1983), Cosmos. Una evolución cósmica de quince mil millones que ha transformado la materia en vida y consciencia. Ed. Planeta. Barcelona.

Spire, A. (2000), El Pensamiento de Prigogine. La belleza del Caos. Ed. Andrés Bello. Santiago de Chile.

Streibel, Michael J. (1989), “Diseño instructivo y aprendizaje situado: ¿es posible el maridaje?” en Revista de Educación, No 289, págs. 215-234.

Zohar, D. (1996), El yo cuántico. Naturaleza humana y conciencia definida por la física moderna. Publimex. Ciudad de México. 